\title{
How Partnership Trust can Facilitate and Result from CBPR: An Assessment of Situational, Organizational, and Institutional Related Factors
}

\author{
Jenneil Charles ${ }^{1 *}$, Arelis Moore de Peralta ${ }^{2}$, Victoria Prieto Rosas ${ }^{3}$ and \\ Julie Smithwick ${ }^{4}$ \\ ${ }^{1}$ Institute on Family and Neighborhood Life, Clemson University, USA \\ ${ }^{2}$ Department of Languages, Clemson University, USA \\ ${ }^{3}$ Programa de Población Facultad de Ciencias Sociales, Universidad de la República, Uruguay \\ ${ }^{4}$ Center for Community Health Alignment, University of South Carolina, USA
}

\section{Research Article \\ Volume 5 Issue 2}

Received Date: March 09, 2021

Published Date: April 02, 2021

DOI: $10.23880 /$ eij-16000186

*Corresponding author: Jenneil Charles, Clemson University, Brackett Hall, Clemson, South Carolina, 29634, USA. Email: jenneic@clemson.edu

\section{Abstract}

This study used secondary qualitative data analysis to determine the extent to which selected constructs of Community-Based Participatory Research (CBPR) promoted and supported the development of partnership trust among organizational and community stakeholders of a community-based health organization (CBHO). The Centers for Disease Control (CDC) identifies partnership trust as an integral factor that contributes to the optimal performance of public health research networks in their attempts to develop relationships with the communities and partners with whom they work. The present study was preceded by two studies which were informed by a modified version of Dietz and Den Hartog's Multidimensional Measure of Trust Model (MMTM). The first study explored perceptions of partnership trust among the organization's stakeholders and informed the development of a bilingual survey instrument (English and Spanish) to measure partnership trust as an outcome of CBPR (CBPR-PTS). The second study evaluated meaning and translation factors and issues related to the CBPRPTS, using cross-cultural cognitive interviewing (CCCI). In the present study we used directed content analysis to evaluate the face validity of the constructs of the "situational, organizational, and institutional constraints" dimension of the MMTM, by analyzing the extent to which the CCCI qualitative data reflected the intended meaning and explanations related to partnership trust development. In addition, we analyzed the extent to which the identified CBPR-related constructs contributed to the development of a trustor-trustee relationship. Findings from this study show that stakeholders' perception of the CBHO's trustworthiness may have been influenced by selected CBPR processes and methods including, (a) facilitating the building of communities' research capacities through collaborative research efforts; (b) sustainability efforts such as empowering community leaders; and (c) facilitating effective communication practices like listening to, learning from, and promoting the participation of the trustors. In addition, that the CBPR-related constructs integrated in the "situational, organizational, and institutional constraints" dimension hold acceptable levels of face validity and are appropriate for use in exploring the role of CBPR in promoting partnership trust. Knowledge gained from our CBPR-PTS development research process has the potential to advance the development of tools to evaluate relevant outcomes of CBPR among underrepresented populations.

Keywords: Partnership Trust; Community-based Participatory Research; Situational, Organizational, and Institutional Constraints; Multidimensional Measure of Trust Model; Latinx; Community Engagement; Health Equity 


\section{Epidemiology International Journal}

Abbreviations: SDOH: Social Determinants of Health; CBPR: Community-based Participatory Research; CBHO: Community-based Health Organization; CDC: Centers for Disease Control; CCCI: Cross-Cultural Cognitive Interviewing; MMTM: Multidimensional Measure of Trust Model; CHW: Community Health Workers; OPs: Organizational Partners; CPs: Community Participants.

\section{Introduction}

Individuals of Latinx ethnicity accounted for $18.1 \%$ of the total United States (US) population as found by the 2017 US Census Bureau [1]. The Latinx population represents the largest minority group in the US and experiences significant health disparities [2]. The leading causes of death for Latinx individuals in the US include cancer, cardiovascular disease, accidents (unintentional injuries), stroke, diabetes, chronic liver disease and cirrhosis [2-5]. Some of the social determinants of health (SDOH) that influence Latinx' health status include structural racism, language barriers, education levels, poverty, household income, decreased access to health care, immigration policy, socioeconomic status, citizenship status, and employment levels [4,5]. As distal variables, these SDOH function as risk factors which have a proximal effect (or impact) that influence the morbidity and mortality of the US Latinx population $[2,5]$. Therefore, the need to address the health inequities that negatively affect Latinx communities in the US is crucial.

Building capacity in Latinx communities has been identified as a key strategy to eliminating health disparities and inequities [6]; particularly, by utilizing culturally appropriate and inclusive research methods and approaches. Community-based participatory research (CBPR) is a collaborative approach to research that has been useful in working with disadvantaged communities to reduce health disparities. CBPR is defined as a collaborative approach to research that, ideally, involves all partners equitably in the research process and recognizes the unique strengths that each brings. CBPR begins with a research topic of importance to the community with the aim of combining knowledge and action for social change to improve community health and eliminate health disparities [7]. Through CBPR, communities have been directly involved in culturally competent research, ranging from the design, implementation and application of culturally centered interventions and findings [8].

$$
\text { One challenge affecting optimal community }
$$

involvement in CBPR has been the adverse experiences that underrepresented and under-resourced communities have experienced historically with researchers and government authorities, resulting in mistrust and suspicion. Community mistrust that results from adverse experiences can strongly influence CBPR partnership development processes [9]. Conversely, CBPR can be used to overcome mistrust toward researchers, as CBPR has been considered as a trust-building process [9]. Therefore, the importance of establishing and fostering trust cannot be overstated in CBPR initiatives [10]. Additionally, developing strong partnership trust among stakeholders is integral to the nature and functioning of community-based health organizations (CBHOs) that are oftentimes partners in CBPR initiatives. The Centers for Disease Control (CDC) identifies partnership trust as an integral factor that demonstrates the performance of public health research networks (networks that include academic researchers, public health agencies, and community members) in their attempts to develop relationships with the communities and other partners with which they work [11]. Partnership trust is a critical determinant of the success of public health research networks [11].

Though the measure of public trust in health care organizations has been studied, the conceptualization and evaluation of public trust in CBPR partnerships is underinvestigated [12]. Gaps remain in how these processes work or how the success of CBPR in fostering trust can be measured [13]. To address this gap, the research team conducted two preliminary studies prior to the one presented in this report, using CBPR methods and Cross-Cultural Cognitive Interviewing (CCCI) (Table 1). The purpose of these studies was to develop and refine a bilingual and quantitative instrument to measure partnership trust as a CBPR outcome $[14,15]$. Both studies were conducted through a communityacademic partnership between Clemson University, the University of South Carolina, and PASOs, a culturally and linguistically informed CBHO that addresses Latinx health disparities through promoting healthy lifestyles and increasing community access to relevant social and health care services; especially among first generation Latinx immigrants whose enhanced vulnerability, paired with their collective and individual experiences, render them to be particularly distrustful of institutions. PASOs' central office is affiliated with the University of South Carolina's Arnold School of Public Health, and its six regional offices are partnered with local health or social service organizations. PASOs' vision is a healthy South Carolina with healthy Latinx communities. 


\section{Epidemiology International Journal}

\begin{tabular}{|c|c|c|c|}
\hline Studies & Phase & Academic partners & Community partners \\
\hline \multirow{3}{*}{$\begin{array}{l}\text { Qualitative pilot } \\
\text { study to assess } \\
\text { and understand } \\
\text { conceptualization, } \\
\text { perceptions and } \\
\text { determinants of } \\
\text { partnership trust, } \\
\text { as an outcome of } \\
\text { CBPR [14]. }\end{array}$} & One & $\begin{array}{l}\text { - Formation of interdisciplinary team } \\
\text { - Design of pilot study } \\
\text { - Team meetings to introduce research team and } \\
\text { the purpose of the pilot study to the PASOs' team } \\
\text { statewide. }\end{array}$ & $\begin{array}{l}\text { - Recruitment of key partners for interdisciplinary } \\
\text { team } \\
\text { - Design of pilot study } \\
\text { - Multiple team meetings to introduce the academic } \\
\text { partner and the purpose of the pilot study to the } \\
\text { PASOs' team statewide. } \\
\text { - Shared development of understanding of the } \\
\text { local context for population served and the multi- } \\
\text { dimensional functionality and frameworks of the } \\
\text { community-based health organization }\end{array}$ \\
\hline & Two & $\begin{array}{l}\text { - Sharing of a literature review to identify contextual } \\
\text { factors that influence partnership trust in CBPR. } \\
\text { - Identification and selection of theoretical } \\
\text { framework (MMTM) to guide the study. }\end{array}$ & $\begin{array}{l}\text { - Discussion of literature findings and suggestions } \\
\text { for additions. } \\
\text { - Selection of theoretical framework (MMTM) to } \\
\text { guide the study. }\end{array}$ \\
\hline & Three & $\begin{array}{l}\text { - MMTM modification by embedding selected } \\
\text { CBPR-processes and methods that could promote } \\
\text { partnership trust in the INPUT section. } \\
\text { - Expanded literature search to identify items used } \\
\text { to assess the MMTM constructs in instruments } \\
\text { developed by others } \\
\text { - Development of focus group and interview } \\
\text { instruments for the pilot study } \\
\text { - Training of a Community Health Worker (CHW) to } \\
\text { assist with interviews } \\
\text { - Coordinated data collection and data analysis } \\
\text { - Co-facilitation of meeting with participants to share } \\
\text { results }\end{array}$ & $\begin{array}{l}\text { - MMTM modification by embedding selected } \\
\text { CBPR-processes and methods that could promote } \\
\text { partnership trust in the INPUT section. } \\
\text { - Revision and validation of additional items to } \\
\text { assess the MMTM constructs. } \\
\text { - Development of focus group and interview } \\
\text { instruments for the pilot study } \\
\text { - Recruitment of CHWs for data collection } \\
\text { - Assistance provided to academic partners in } \\
\text { - Coordinating data collection } \\
\text { study participants to share results and mediated } \\
\text { discussion about importance of the study and } \\
\text { their roles in it }\end{array}$ \\
\hline \multirow{3}{*}{$\begin{array}{l}\text { Using Cross- } \\
\text { cultural Cognitive } \\
\text { Interviewing } \\
\text { (CCCI) to refine } \\
\text { a culturally/ } \\
\text { linguistically } \\
\text { relevant } \\
\text { instrument } \\
\text { to measure } \\
\text { partnership trust } \\
\text { in the context of } \\
\text { CBPR [15]. }\end{array}$} & Four & $\begin{array}{l}\text { - Expansion of interdisciplinary team (two additional } \\
\text { academic partners). } \\
\text { - Working meetings to develop a bilingual (English } \\
\text { and Spanish) quantitative instrument to measure } \\
\text { CBPR partnership trust } \\
\text { - Translation and back-translation of quantitative } \\
\text { instrument }\end{array}$ & $\begin{array}{l}\text { - Working meetings to develop a bilingual (English } \\
\text { and Spanish) quantitative instrument to measure } \\
\text { CBPR partnership trust } \\
\text { - Review of translation and back-translation of } \\
\text { instrument }\end{array}$ \\
\hline & Five & $\begin{array}{l}\text { - Facilitation of pilot testing of new quantitative } \\
\text { instrument } \\
\text { - Instrument's adaptation based on pilot testing } \\
\text { results } \\
\text { - Coordinated adaptation of the survey to different } \\
\text { types of stakeholders' role and degree of } \\
\text { involvement with the organization }\end{array}$ & $\begin{array}{l}\text { - Recruitment of stakeholders for pilot testing } \\
\text { based on trust already built between CBHO and } \\
\text { community members } \\
\text { - Instrument's adaptation based on pilot testing } \\
\text { results } \\
\text { - Assisted with adaptation of the survey to } \\
\text { different types of stakeholder roles and degrees } \\
\text { of involvement with the organization }\end{array}$ \\
\hline & Six & $\begin{array}{l}\text { - Development of CCCI questionnaire. } \\
\text { - IRB protocol development, submission and approval } \\
\text { - Recruited staff CHWs and organizational partners } \\
\text { - Administration of survey and interviews } \\
\text { - Data analysis }\end{array}$ & $\begin{array}{l}\text { - Assisted with development of CCCI questionnaire } \\
\text { - Recruitment of volunteer CHWs and community } \\
\text { participants } \\
\text { - Data analysis }\end{array}$ \\
\hline
\end{tabular}

Table 1: Depiction of the research process of a community-academic partnership to develop a culturally/linguistically relevant survey instrument to measure partnership trust as a CBPR outcome: Two preliminary studies. 


\section{Epidemiology International Journal}

The two antecedent studies depicted in table 1 , as well as the present study described here represent the interdisciplinary research team's efforts to address the lack of valid quantitative assessment tools to assess partnership trust as a CBPR outcome by developing the CBPR Partnership Trust Survey (CBPR-PTS). The research team considered Dietz G, et al. [16] Multidimensional Measure of Trust Model (MMTM) as appropriate to inform development of the CBPR-PTS because it conceptualized trust as a process that requires inputs (CBPR related concepts in our modified version) which catalyze a progression that results in trust as the output (i.e. certain inputs allow for the development of trust). Dietz G, et al. posited that the inputs in the model allow for the progression of trust as a belief, then as a decision and ultimately as an act; thus, resulting in trust as an action is the output [16]. In the present study the team evaluated the face validity [17] of one of the input dimensions of a modified version of the MMTM labeled as "situational, organizational, and institutional constraints" (Figure 1).

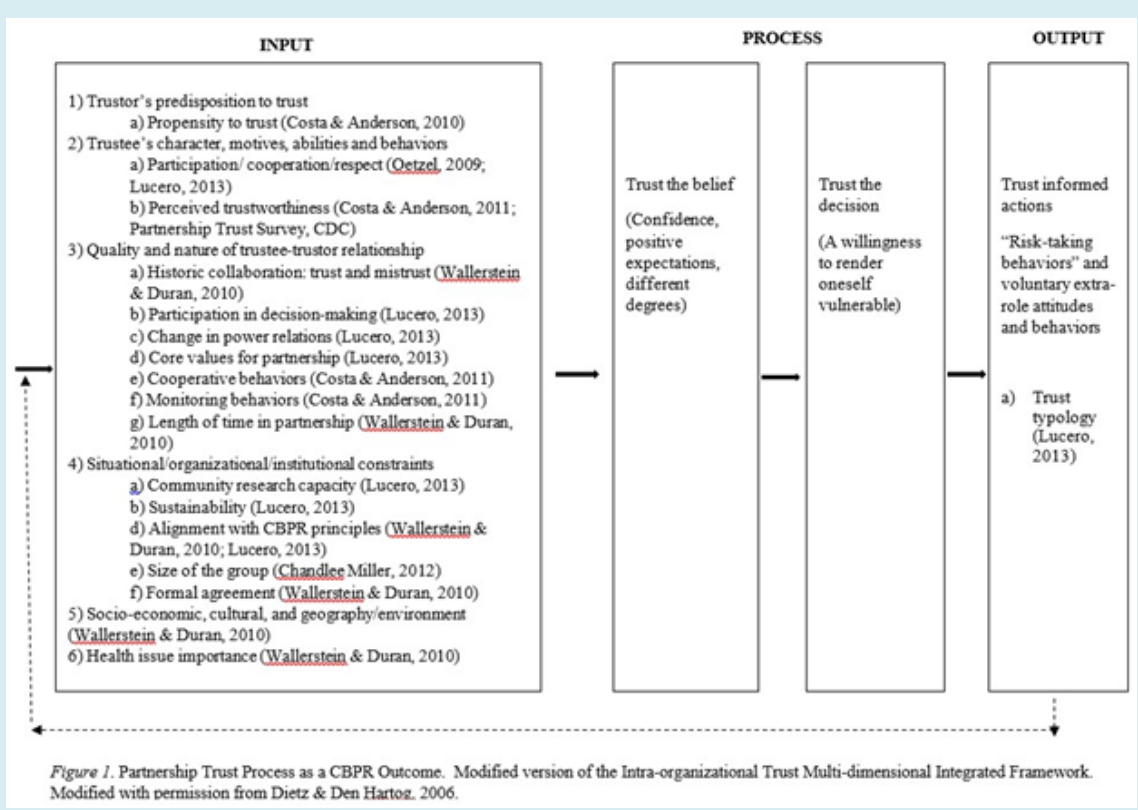

Figure 1: Partnership Trust Process as a CBPR Outcome, Modified version of the Intra-organizational Trust Multi-dimensional Integrated Framework, modified with permission from Dietz G, et al. [16].

In the first study (Table 1), the research team conducted a literature review to modify the MMTM by incorporating key CBPR principle-related constructs in its input dimensions (Figure 1). The constructs identified represented contextual factors or facilitators of partnership trust development in community-engaged research and interventions for reducing health disparities in underrepresented groups. The modified MMTM incorporated constructs from the following studies: a study about types and perceptions of trust, as well as relevant factors that influence trust development in the context of CBPR [9]; the development of the CBPR Conceptual Logic Model [18]; formative and reflective indicators of team trust [19]; the exploration of trust, group characteristics and health among community-based and congregation-based groups [20]; the identification of intercultural communication concepts [21]; and, the CDC's partnership trust survey [11].

To orient the design of the present study to evaluate face validity [17] of the input dimension of "situational, organizational, and institutional constraints" of the
MMTM, the research team conducted a literature review to identify approaches previously used for framework evaluation. We found that researchers have used costeffectiveness analyses to evaluate frameworks [22,23], as well as [24] frameworks used to develop care coordination interventions and programs. A study conducted by Belone L, et al. [17] presented how the authors sought community input to assess face validity and acceptability of a CBPR framework through semi-structured focus groups with six partnerships nationwide. In our study, we analyzed qualitative data, gathered through interviews, to conduct a subjective assessment of whether the constructs in the input dimension of "situational, organizational, and institutional constraints" holds face or conceptual validity by reflecting the meanings related to the MMTM measures that it is supposed to measure. The research question that guided this study was, to what degree does the CBPR-related constructs incorporated into the dimension of "situational, organizational, and institutional constraints" of a modified version of the Multidimensional Measure of Trust Model 


\section{Epidemiology International Journal}

(MMTM) account for the influence of CBPR processes and methods in promoting partnership trust?

\section{Methods}

\section{Setting}

The interdisciplinary team's research on partnership trust has been facilitated by a community-academic partnership between Clemson University, the University of South Carolina and PASOs. This ongoing collaboration began in 2010. In 2014, the partners agreed to build on their partnership by addressing the existing gap in the availability of innovative quantitative measurement tools for community engaged research's outcomes by conducting the two studies that were described in the introduction section of this publication.

PASOs, which translates into "steps" in English, is a CBHO that serves Latinx communities in South Carolina, by providing education, family health and early childhood resource navigation and advocacy in a manner that is culturally responsive and relevant to the population they serve; while also taking into account the sensitivity required in serving the needs of majority first-generation immigrants whose vulnerabilities and experiences of systemic racism have caused them to be distrustful and less willing to engage in help-seeking behaviors from traditional institutions. PASOs' organizational model is likened to a public health research network; with its intentional focus on public health, the organization provides pathways that connect community members to other organizational partners, which can include public health agency representatives and academic researchers. Therefore, PASOs directs participants to culturally appropriate resources when they are in need. This is typically initiated through Community Health Workers (CHWs) who are grassroots leaders within the communities that PASOs serve, and who serve as facilitators between Latinx communities that need increased access and the organizations that have services that the communities need. PASOs' model is grounded in Latin American cultures. The trainings that the CHWs participate in are culturally tailored, building on participants' strengths and experiences, and giving information and skills related to accessing needed resources, advocating for increased equity, and providing culturally appropriate outreach.

Furthermore, to address the lack of capacity that exists among many organizations to provide culturally responsive services, and to begin to ameliorate the effects of structural racism, PASOs engages in capacity building with health care and social service providers. Through these partnerships, PASOs helps organizations and their leaders to better understand and more adeptly cater to the needs of the Latinx communities they serve and build better networks among the stakeholders who interact with the services that organizations provide. Methods that PASOs employs to build the capacities of these organizations include assessments, cultural competency trainings, ongoing technical support and constructing strategic plans with measurable goals. PASOs also frequently contribute to and facilitate qualitative research, including CBPR, with the communities they serve. These efforts are in line with their mission statement, which states, "PASOs helps build a stronger South Carolina by supporting Latino communities with education, advocacy, and leadership development."

\section{Sample}

The sample for the CCCI study, from which data analyzed for this study was obtained, consisted of PASOs' stakeholders. Clemson University's IRB approved the CCCI study. Twenty-one stakeholders completed the CBPR-PTS and were interviewed using CCCI as follows: community participants or clients (CPs, $n=5)$, volunteer Community Health Workers (CHWs) $(n=5)$, organizational partners (OPs, $n=6)$, and staff CHWs $(n=5)$. Table 2 includes the socio-demographic characteristics of interview participants. Related to partnership trust, the trustors included community participants and organizational partners, while PASOs' staff and leaders were the trustees. The volunteer CHWs occupy a unique position in this relationship because they mediate between PASOs, CPs, and OPs; therefore, they represent both trustors and trustees.

\begin{tabular}{|c|c|c|c|c|c|}
\hline Stakeholder category & $\begin{array}{c}\text { Surveyed in } \\
\text { Spanish }\end{array}$ & Female & $\begin{array}{c}\text { Latinx/ } \\
\text { Hispanic }\end{array}$ & $\begin{array}{c}\text { Foreign- } \\
\text { born }\end{array}$ & $\begin{array}{c}\text { Total number of } \\
\text { stakeholders }\end{array}$ \\
\hline Staff CHWs & 2 & 4 & 4 & 3 & 6 \\
\hline Community Participants/ Clients (CP) & 4 & 4 & 4 & 4 & 4 \\
\hline Volunteer CHWs & 5 & 5 & 4 & 5 & 5 \\
\hline Organizational Partners (OP) & 0 & 3 & 4 & 4 & 6 \\
\hline Total & 11 & 16 & 16 & 16 & 21 \\
\hline
\end{tabular}

Table 2: Demographic characteristics of partnership trust survey and CCCI participants $(n=21)$. 


\section{Epidemiology International Journal}

Some interview questions had to be adjusted by considering the differences among stakeholders' characteristics and experiences (i.e., roles and degrees of involvement in PASOs, experience with academic research, and with program design). The interview questions were based off the items in the original CBPR/PTS and aimed to address any misunderstandings and confusion about the wording of the survey questions (Table 3). A more detailed explanation about the CCCI [25] data collection and analysis procedures can be found in Moore de Peralta, et al. [15].
MMTM Dimensions*

\begin{tabular}{|l|}
\hline \multicolumn{2}{|c|}{ MMTM Dimensions* } \\
\hline $\begin{array}{l}\text { 1) Trustor's predisposition } \\
\text { to trust }\end{array}$
\end{tabular}

2) Trustee's character, motives, abilities and behaviors

\section{Focus groups and interviews questions}

\section{Input}

1. What is the first thing that comes to mind when you hear the word PASOs? [Prompt: What kind of words come to your mind when you hear someone mention PASOs? If you had to describe PASOs as an organization, what would you say?]

4. (a) What kinds of people do you work with in PASOs, including staff, other organizational partners, and community health workers? [Prompt: If any stakeholder is excluded from participants' responses, please mention it]

(b) What influences how you work with PASOs staff? With other organizational partners? And with community health workers?

3) Quality and nature of trustee-trustor relationship

2. How do you describe your relationship with the organization PASOs?

7. (a) Does it make a difference that some of the PASOs team members and coordinators either

4) Situational, organizational, and institutional constraints

are Hispanics or had a lot of experience working with the Hispanic community? [Prompt: We refer to a difference in the effectiveness or reach of the program into the community]

(b) If so, why? [Prompt: If this question is answered in question a, jump to question 8]

5) Socio-economic, cultural, and environment

N/A

6. (a) Do you have an idea of what PASOs does? (b) Are there any other health topics that you

6) Health issue importance think are important and PASOs should be also addressing? [Prompt: This question refers to health topics like prenatal health or chronic disease prevention]

\section{Process}

Trust the belief: confidence positive expectations.

3. In your own words, how do you define trust in what happens in your work with or participation in PASOs? [Prompt: Trust can be expressed in relation with the trust in your family, your bank, in your friends, etc. However, I would like for you to refer to your trust in PASOs.]

5. (a) In general, how would you describe your level of trust in PASOs staff you worked with? How about with other organizational partners you worked with in PASOs? And in the community health workers you worked with in PASOs?

Trust the decision: (a willingness to render oneself vulnerable) Trust Typology (Lucero, 2013)

[Prompt: This question refers to if you have trust in these stakeholders, and if this is the case, is this a total trust, some trust, or no trust?]

(b) How has your trust level changed over time? [Prompt: Your trust might have changed to having more trust, less trust, or no changes in your level of trust]

(c) What do you think made your trust change?

[If they answer c in question b, skip c]

\section{Output}

Trust informed actions: "Risk-taking behaviors" and voluntary extra-role attitudes and behaviors

8. (a) Have community health workers and/or other community members been involved in the work PASOs has done with your organization? [Prompt: We refer to involving them in planning/ improving your work, methods, outreach]

(b) What do you think about this involvement? [Prompt: If this question is answered in question a, then jump to question 9]

*please refer to Figure 1 for detailed visualization of the relationships among the MMTM dimensions and processes, as well as the source from which these constructs were obtained.

Table 3: Data collection instrument questions according to conceptual framework (Multi-dimensional Measure of Trust Model [MMTM], Modified from Dietz G, et al. [16]. 


\section{Epidemiology International Journal}

\section{Data Analysis}

This study represents a secondary qualitative data analysis approach using the CCCI data collected in the second preliminary study. We used directed content analysis [26] to evaluate the face validity of the constructs of the dimension of "situational, organizational, and institutional constraints" of the MMTM, by analyzing the extent to which the CCCI qualitative data reflect meaning and explanations on how CBPR promotes partnership trust development among PASOs stakeholders. In addition, we analyzed the extent to which the identified CBPR-related constructs contribute to the development of a trustor-trustee relationship.

Hsieh $\mathrm{H}$, et al. [26] method of directed content analysis involves the use of predetermined codes. The research team identified the predetermined codes by analyzing the CBPR constructs from selected sources included in the literature review previously conducted by the team to modify the MMTM. From this literature, we included codes from publications of Chandlee Miller M [20], Lucero JE [9], and Wallerstein $\mathrm{N}$, et al. [18]. We subjected these predetermined codes to each one of the constructs (i.e., community research capacity, sustainability, alignment with CBPR principles, size of the group, and formal agreement) included in the dimension of "situational, organizational, and institutional constraints" of the modified MMTM model. The purpose was to uncover the degree to which the data aligns with the constructs of one of the dimensions of the modified MMTM. The actions taken to conduct the directed content analysis are documented in Table 4.

\begin{tabular}{|c|c|c|}
\hline Steps & Hsieh \& Shannon Process & Data Analysis Approach \\
\hline Part 1 & $\begin{array}{l}\text { Deductive category application; using the existing } \\
\text { framework to provide predictions about the variables of } \\
\text { interest or about the relationships among variables, and } \\
\text { then determine the initial coding scheme or relationships } \\
\text { between codes }\end{array}$ & $\begin{array}{l}\text { Hypothesis: The CBPR constructs that Moore de } \\
\text { Peralta, et al. [15] embedded into the dimension } \\
\text { of "situational, organizational, and institutional } \\
\text { constraints" of the MMTM should function as } \\
\text { facilitators or promoters of partnership trust. }\end{array}$ \\
\hline Part 2 & $\begin{array}{l}\text { Coding; can begin with one of two strategies, depending on } \\
\text { the research question. } \\
\text { Strategy 1; the goal of the research is to identify and } \\
\text { categorize all instances of a particular phenomenon } \\
\text { through: a. reading the transcript and highlight all text that } \\
\text { on first impression appears to represent the phenomenon } \\
\text { b. coding all highlighted passages using the predetermined } \\
\text { codes. Any text that could not be categorized with the initial } \\
\text { coding scheme would be given a new code. } \\
\text { Strategy 2; begin coding immediately with the } \\
\text { predetermined codes. Data that cannot be coded are } \\
\text { identified and analyzed later to determine if they represent } \\
\text { a new category or a subcategory of an existing code. }\end{array}$ & $\begin{array}{l}\text { Strategy } 1 \text { was used; the first author consulted } \\
\text { selected literature from the one used by Moore } \\
\text { de Peralta, et al. [15] to identify and incorporate } \\
\text { CBPR constructs into the dimension of "situational, } \\
\text { organizational, and institutional constraints" of the } \\
\text { MMTM. Instances for each construct were identified } \\
\text { and categorized - themes that emerged from the } \\
\text { discussions/explanations of the constructs in the } \\
\text { literature were codified and are presented in Table } \\
\text { 5. }\end{array}$ \\
\hline Part 3 & $\begin{array}{l}\text { The findings from a directed content analysis offer } \\
\text { supporting and non-supporting evidence for a theory. This } \\
\text { evidence can be presented by showing codes with exemplars } \\
\text { and by offering descriptive evidence. }\end{array}$ & $\begin{array}{l}\text { The findings from the directed content analysis } \\
\text { were analyzed to determine whether the CCCI data } \\
\text { offered supporting and non-supporting evidence } \\
\text { for the face validity of the constructs embedded } \\
\text { in the dimension of the dimension of "situational, } \\
\text { organizational, and institutional constraints" of the } \\
\text { modified MMTM [16] in determining partnership } \\
\text { trust among PASOs stakeholders. }\end{array}$ \\
\hline
\end{tabular}

Table 4: Table depicting the process involved in directed content analysis according to Hsieh \& Shannon [26]. 


\section{Epidemiology International Journal}

\section{Results}

"Situational, organizational, and institutional constraints" represents one of the four contextual dimensions of trust in the original Dietz and Den Hartog Multi-dimensional Measure of Trust Model (MMTM) [16]. This dimension considers those organization-related processes and methods that influence the trustor's decision whether to trust the organization. The results are presented in accordance with the modified MMTM's CBPR-related constructs of this dimension including community research capacity, sustainability, alignment with CBPR principles, size of the group, and formal agreement. The variables or categories, identified in a literature review and used to code the CCCI data, are listed under each CBPR-related construct of the dimension (Table 5). It should be noted that the framing of the results, in conjunction with the codes used in this study, allows for overlap across constructs regarding the role CBPR plays in determining partnership trust among PASOs' stakeholders.

\begin{tabular}{|c|c|}
\hline $\begin{array}{l}\text { CBPR Construct (MMTM's "situational/ } \\
\text { organizational/ institutional constraints" } \\
\text { dimension) }\end{array}$ & Codes \\
\hline Community research capacity [9] & $\begin{array}{l}\text { - Skills and expertise } \\
\text { - Data and information } \\
\text { - Legitimacy and credibility } \\
\text { - Ability to bring people together for meetings and activities } \\
\text { - Connections to relevant stakeholders }\end{array}$ \\
\hline Sustainability [9] & $\begin{array}{l}\text { - Long-term commitment } \\
\text { - Expressions of obligation and dedication } \\
\text { - Evaluation of funding needs }\end{array}$ \\
\hline Change in communication level outcomes [9] & $\begin{array}{l}\text { - Listening (as facilitator of respect and understanding) } \\
\text { - Learning (as facilitator of creating shared meaning...bridging } \\
\text { differences) } \\
\text { - Participation (as facilitator to familiarity; sharing knowledge, skill and } \\
\text { resources) } \\
\text { - Commitment (performance of shared values [action]; demonstration } \\
\text { of "being in this together") }\end{array}$ \\
\hline $\begin{array}{l}\text { Alignment with CBPR principles }[18,9] \text {. } \\
\text { (a) Genuine partnership means all parties, } \\
\text { academic and community partners, learn from } \\
\text { each other } \\
\text { (b) Research efforts include capacity building (in } \\
\text { addition to conducting the research, there is a } \\
\text { commitment to training community members in } \\
\text { research) }\end{array}$ & $\begin{array}{l}\text { (a) Genuine partnership (understood in terms of): } \\
\text { co-learning [informal and formal], mutual knowledge/expertise's } \\
\text { recognition, and teaching } \\
\text { (b) Capacity building (understood in terms of): } \\
\text { community members' participation in research training and } \\
\text { implementation }\end{array}$ \\
\hline Size of the group [20] & Preference for small groups \\
\hline Formal agreement [18] & $\begin{array}{l}\text { - Verbal or written agreement for collaborative efforts } \\
\text { - Formal agreements on roles and responsibilities }\end{array}$ \\
\hline
\end{tabular}

Table 5: Table listing the codes derived from the literature review used in the directed content analysis of the cross-cultural cognitive interviews (CCCI).

\section{Community Research Capacity}

According to Lucero JE [9], the community's capacity for research is one of the elements related to trust in research conducted through partnerships between the community and health organizations. The codes identified by the research team to analyze the extent to which the CBPR construct of community research capacity aligns with the MMTM were, skills and expertise, data and information, legitimacy and credibility, ability to bring people together for meetings and activities, and connection to relevant stakeholders [9].

Skills and expertise: Partnership trust develops among PASOs' stakeholders when the skills and expertise of each stakeholder are taken into consideration. Community participants (CPs), sometimes referred to as "clients" in 


\section{Epidemiology International Journal}

other organizations, are able to develop trust in PASOs' leadership and staff as a belief, a decision and an act; not only when they believe in and depend on their skills and their expertise, but also when their own skills and expertise are integrated into PASOs' research and program development. When participants can share intimate knowledge about their community (such as strengths, needs, culture, norms and values), that knowledge can be used to benefit both the organization and the community at large. As one CP said, "PASOs wouldn't be able to know what kind of help to provide without the community telling them." However, PASOs' leadership and staff could potentially compromise the development of partnership trust if community participants' expertise is not validated due to lack of follow-up or updates regarding the information that they have shared with PASOs, or if PASOs' staff and leadership does not express value in CPs' knowledge and expertise. The value of validation as it contributes to building partnership trust and confidence is reflected in a volunteer CHW's recounting of being involved in PASOs' decision-making process by saying, "It makes me feel very proud when I am able to contribute a small grain of sand, but when it's heard and used, it's when you really can tell that it is effective and that it works, and you think WOW, that was all due to my contribution."

Just as CPs rely on the skills and expertise of PASOs' representatives (staff and volunteer Community Health Workers-CHWs) to determine whether to trust the organization, volunteer CHWs also rely on PASOs' staff CHWs and Organizational Partners (OPs) to help them cultivate their skills and expertise to serve their communities better. Volunteer CHWs also rely on opportunities for involvement in PASOs' research and programs to build their capacity and expertise in serving their communities. In developing their skills, expertise and capacity, CPs can trust volunteer CHWs in the positions they fill to assess and provide for the needs of their communities as evidenced by a volunteer CHW's discussion of how working with PASOs has enabled her to really help her community:

"...[W] hen you become part of groups like these you learn about how you can help others, especially children, and not spend so much time alone at home. You come into direct contact with families and visit their homes, you learn more about them and their needs. This motivates me to keep helping others. Some people are scared to leave their homes because they do not have the tools to find a lawyer or legal documents, afraid the authorities will catch them, and [they] learn to trust me."

Volunteer CHWs' skills and expertise also allow CPs to trust them enough to participate in the research and workshops they facilitate on behalf of PASOs. PASOs' staff CHWs develop their capacity to be trustworthy by developing skills and expertise in collaborating with external partners, as well as, in data analysis. Staff CHWs' process of incorporating communities' expertise and intimate knowledge in PASOs' research and programming also contributes to CPs' and volunteer CHWs' development of trust in staff CHWs. Consulting CPs' needs and concerns also influences OPs' disposition to trust in PASOs. For example, an OP stated, "[it's] important that they are hearing from the community what the needs are versus what they think the needs are."

Data and information: The CCCI data showed that sharing data and information is instrumental to developing partnership trust within a CBPR relationship. The way data and information are collected, as well as how this information is used and the effects of the aftermath of this usage, all influence partnership trust. Volunteer and staff CHWs collect data and information provided by the community as a means of gaining greater understanding of the strengths and needs of the community. For example, a volunteer CHW explained, "...much of what we gather in the community allows us to adjust the programs or the proposals, the needs of the people, the schedules, the possibilities and the language, the common language that one uses, or the activities that I assigned." Staff and volunteer CHWs also collect information from CPs to gain insight into the nuances of the community, which is used to help improve the delivery of programs and services to the community. CPs' expectations that the staff and volunteer CHWs will use the data collected to benefit their community influences their trust in the organization. In this same way, PASOs' leadership gains volunteer $\mathrm{CHWs}^{\prime}$ trust by using the information they collected to build on the strengths and provide for the needs of the community. CPs also develop partnership trust by being involved in the data collection process. On a primary level, they are invited to participate in the research process through recruiting fellow community members to participate, and participating in the evaluation/analysis phase of PASOs' investigations, etc. This collaborative effort with other stakeholders involved in data collection fosters a greater inclination for CPs to believe, decide, and act on trust in the partnership. OPs are also inspired to develop trust in their partnership with PASOs upon having the opportunity to work collaboratively with CPs.

Legitimacy and credibility: Trustworthiness is also dependent on the legitimacy and credibility of the information that has been collected for research or programmatic purposes. PASOs will not be able to deliver programs and services that are customized to the prevalent strengths, needs and cultures of the communities they serve if legitimacy and credibility have not been established. Therefore, staff and volunteer CHWs work to ensure that their data and information are up-to-date, especially by means of research. A staff CHW said, "[w]e see it as compiling data... 


\section{Epidemiology International Journal}

if when we bring participants in, we will connect them with multiple pathways on things to remedy whatever situations they may need. We track that and we have follow-ups. So, at the end of the day, at the end of each month, we know exactly how many of what we've done." In discussing the role of research, a volunteer CHW viewed it as, "an investigation task [where] you have the population and then you have to ask a lot of questions....and then you have to evaluate and make sense of it all..." Thus, evoking how essential research is to establishing legitimacy and credibility in helping PASOs aptly discern the needs of the population they serve. Furthermore, PASOs' leadership assesses the legitimacy and credibility of their programs and services by conducting program evaluations and using the information provided by the community participants (CPs) to crosscheck the integrity of their programs. CPs also contribute to the legitimizing of the programs by validating and sharing up-to-date information with PASOs staff and volunteer CHWs.

Ability to bring people together for meetings and activities: Being active in collaborative research based on a CBPR model allows the PASOs team to exercise their abilities to bring communities together. Volunteer CHWs recruit CPs for PASOs' research projects and workshops. Staff CHWs rely on their volunteer CHWs' ability to recruit and bring community members together to engage in research. Bringing people together for CBPR purposes and other PASOs programs presents opportunities to ingrain equity in the partnership because it "promotes an empowering and power-sharing process that attends to social inequalities" [9].

Connections to relevant stakeholders: Volunteer CHWs serve as key mediators between CPs, and staff CHWs and OPs. As volunteer CHWs are CPs that have been trained by PASOs, their mediating role helps promote partnership trust between CPS and the organization. Volunteer CHWs are PASOs' most direct and organic connection to the communities they serve. Volunteer CHWs are also connected to OPs through programmatic collaborative efforts with community-based and national organizations, and in research efforts with academic partners. Due to their unique role, volunteer CHWs need to trust OPs and staff CHWs to collaborate with them in providing for the needs of the community; while CPs rely on volunteer CHWs to connect them to resources they need.

\section{Sustainability}

Within the context of collaboration among public health research networks and the CBPR process, sustainability is highly prioritized, because it facilitates the development of and commitment to partnership trust among the stakeholders. In other words, when it comes to collaboration among stakeholders in the CBPR relationship, sustainability functions as an outcome of CBPR research and as a facilitator of partnership trust in the relationship [9]. Therefore, based on Lucero JE [9] conceptualization of sustainability, the codes used for this construct include long-term commitment, expressions of obligation and dedication, and evaluation of funding needs, to investigate the extent to which CBPR promotes partnership trust among PASOs' stakeholders.

Long-term commitment: For partnership trust to be sustained in a CBPR relationship, stakeholders need to maintain a long-term commitment. Volunteer CHWs expressed that although they would like to commit longterm to PASOs, other responsibilities, financial restrictions, and lack of enough monetary compensation impinge on their ability to commit. For example, a volunteer CHW said, "I honestly do not know right now. I would love to, but I have other responsibilities like school and family, and I cannot abandon one to focus on another." Given that volunteer CHWs are considered mediators between CPS with staff CHWs and OPs, this inability to commit long-term can potentially compromise the maintenance of partnership trust among various stakeholders. However, those who choose to continue volunteering considering these challenges, especially the absence of significant monetary compensation, demonstrate their long-term commitment. Staff CHWs also face impediments to committing long-term to PASOs.

The organization's reliance on short or medium-term grants and contracts presents the challenge of staff rotations. The nature of grant-funded work makes it difficult for staff members to commit long-term, which can potentially affect how volunteer CHWs are able to trust PASOs to help them serve communities. Nevertheless, many staff members are committed to working at PASOs for as long as circumstances permit, due to their passion for the work and mission. Furthermore, OPs expression of long-term commitment can help ameliorate the concerns of staff CHWs. Some OPs have outwardly expressed their long-term commitment to PASOs, while some have even embedded PASOs' work into their organization. The OPs' commitment evidences their willingness to trust PASOs' partnership. One OP said, "[y] ou know, we have been funding them for such a long time that we have a plan to continue our partnership." Another OP said, "I am 68 years old and I'm choosing not to retire because I have too much interest and energy to give so I'll be a part of PASOs as long as [the executive director] allows me to be."

Expressions of obligation and dedication: Volunteer CHWs expressed many instances of obligation and dedication to PASOs that reflect their role as trustor and trustee. Volunteer CHWs' sensitivity to their communities' vulnerabilities evoked a sense of dedication. Volunteer 


\section{Epidemiology International Journal}

CHWs expressed a willingness to modify the delivery of programs in efforts to facilitate the engagement of their communities. This program modification includes adjusting schedules and curricula to better accommodate community members and amending the goals of the program to enable PASOs to execute its mission more efficiently as it relates to serving communities. In keeping with being sensitive to the communities' vulnerabilities, volunteer CHWs sometimes go beyond their duties to cater to the needs of community members and they also find ways to facilitate smaller-scale programs and services requested by community members. PASOs' staff CHWs support and routinely check in on the volunteer CHWs, which demonstrates dedication from leadership. These acts of obligation and dedication help CPs develop trust in volunteer CHWs, and by extension, in staff CHWs and other PASOs' leaders.

Staff CHWs also exhibit expressions of obligation and dedication that allow them to be trustworthy trustees in the CBPR relationship. In their relationship with the communities they serve, staff CHWs demonstrate obligation and dedication by cultivating and investing in natural leaders in the community. A staff CHW shared that, "[o] ur model is to find those natural leaders in the community and then give those more tools and strengthen their ability to connect." Priming these leaders in advocacy benefits the communities they represent in the end. Staff CHWs are also receptive to being adaptable, with the intention of suitably meeting community needs. Their competence in adaptability is contingent upon the use of data and relevant information in their decision-making processes. Rather than engaging in lackluster or one-sided decision-making processes that are mostly opinion-based, they rely on and pay close attention to, pertinent details informed by the communities they serve. An example of PASOs' obligation and dedication was illustrated by their use of a more family-oriented approach to the delivery of their programs and services based on information that CPs shared with them about the central role that the family has in Latinx cultures. A staff CHW echoed the emphasis of PASOs' family-oriented approach by saying,

"The importance of family comes through in all the programs. In that, you know one of the central focuses is to help families have the resource they need to be able to be stronger. Also recognizing how important family is in people's lives. So even when planning an event or programs, you consider that the person has a family. You know, and so they provide childcare for moms to bring their kids or include the partners or husbands in events when they could. Just recognizing that a person is part of a family and that is what matters."

PASOs' OPs have also demonstrated expressions of obligation and dedication, which indicates their trust in
PASOs as partners. One of the OPs explained how they made policy changes in the way their organization operates based on information gained from their relationship with PASOs. Those changes have long-lasting impacts that will continually benefit the communities to which the policies apply. Staff CHWs maintain that they work to create an environment that embraces and facilitates open communication. OPs have expressed their dedication to the open communication process to ensure that PASOs' mission is being executed through their partnership. OPs also feel obligated to learn from the communities they work with through PASOs because their input also helps improve the services that they provide. As one of the participant OPs shared, "...[W] hat comes to mind is that whenever we've been to a meeting and when the topic would be specific to children with risk factors that we deal with in parenting programs, I could participate and speak up and even speak up and say 'oh gosh I don't understand this risk factor in terms of the Hispanic population.' We are talking about alcoholism or drug abuse, '... is this as rampant in your populations as it is in with what I deal with in the Black population?' It's nice to have those kinds of conversations to help me and [my early childhood organization] know how to better serve a population that I did not know personally till I became a part of PASOs."

Evaluation of funding needs: This variable primarily plays a role in the trustor-trustee relationship between PASOs and their Organizational Partners (OPs). Some OPs provide funding to PASOs, as trustors, based on the PASOs team's capabilities to deliver certain outcomes that are based on communities' needs. A staff CHW explained, "[a] lot of times we would identify a need in the community and study it a little bit. Sometimes a survey or community involvement... from partners to community participants who might want to do this. We secure funding, usually through a grant. Through that grant money we have certain outcomes, certain things we'd have to do, you know, to show [we] were doing this program and what was needed..." Therefore, as trustees, PASOs is expected to follow through with the deliverables stipulated by the OPs. Thus, in order to do so, PASOs must assess the communities' needs and apply for funding that will allow them to address the needs. As trustors, the OPs believe that PASOs has demonstrated the capacity to fulfill the expectations set in the funding contract and meet the needs of the Latinx communities in a culturally appropriate way.

\section{Change in Communication-Level Outcomes}

Lucero JE [9] discussed how acts of communication are essential to the process and progress of CBPR. In noting the vital role of communication, Lucero proposed that these acts also play a role in developing partnership trust, by facilitating the participation of each stakeholder in 


\section{Epidemiology International Journal}

decision-making processes, establishing a sense of mutual understanding of each other's backgrounds and values, and facilitating the exchange and acquisition of information. These features contribute to developing partnership trust by means of determining trustworthiness among the stakeholders; thus, observing changes in communicationlevel outcomes throughout the CBPR process provides insight into the development of partnership trust among PASOs stakeholders. The codes used in this study to determine how changes in communication-level through CBPR affected the development of partnership trust among PASOs stakeholders, included factors such as listening, learning, participation and commitment.

Listening: Within the context of CBPR, listening facilitates respect and understanding among those involved in the relationship [9]. Concerning partnership trust, respect and understanding are instrumental inputs towards developing trust. When CPs involved with PASOs feel respected and understood, this can prompt willingness to be vulnerable with the PASOs team, and confidence in positive expectations when deciding to trust PASOs. When CPs feel respected and understood, they perceive the organization as more trustworthy. This sense of respect and understanding is developed when PASOs gathers feedback from community members and there is a collaborative effort between both stakeholders in deciding how assistance will be given and received.

In addition to being Latinx immigrants themselves, or children of immigrant parents, CHWs have acquired greater understanding of the distinctive concerns that are specific to the communities they serve by adhering to an open-communication approach, which enhances a feeling of mutual respect and understanding. Their shared cultural elements, as well as this cognizance, makes it possible for volunteer CHWs to both understand shared experiences and acknowledge diversity within Latinx cultures, incorporating considerations of this diversity into the planning and delivery of programs and services. It also allows volunteer CHWs to be more understanding of the hardships that Latinx immigrants face when they relocate to the US, allowing them to be more aware of the social and other determinants influencing their communities. A volunteer CHW exemplified this by saying,

"[y]es, in one of my programs I have given my views on how to adjust the program. I have been given that opportunity, not to keep a curriculum that they gave me and deliver it as it was given, but I have been allowed my own inspiration based on the experience with the people, of making it simpler, and that reaches everyone. In my group, there are women that don't read, and ladies who are more educated and that have more schooling. And it's up to me to modify the programs to reach everyone with the same message; they have facilitated me to do that."

PASOs, as an organization, also contributes to partnership trust development by respecting the unique knowledge CHWs have of the CPs they work with. As a result, CHWs are able to make changes in the programming due to the responsive nature of PASOs' modus operandi. Furthermore, the organization increases its capacity to be trustworthy by its emphasis on proactive teamwork. A staff CHW shared an example of how the team demonstrated proactive teamwork when reconstructing the curriculum for one of PASOs' programs. This staff CHW said,

"[w]e were coming up with the themes and curriculum on the health subjects for PASOs. It wasn't just me; it was the entire team at that time that worked together on it, now we have a lot of newer people. But back then the entire team would collaborate on the ideas and ultimately created the curriculum. (...) Yes, contributions are always valued at PASOs. Everyone's opinions and contributions matter."

Learning: Although learning as a variable corresponds with community research capacity, it also represents a specific function in terms of changing communication level outcomes. Within the context of CBPR processes and methods that influence partnership trust, learning (in relation to change in communication outcomes) functions as a facilitator of creating shared meaning and bridging differences among those involved in the relationship [9]. Learning is conducive to developing partnership trust because the partners feel as though their perspectives are "welcomed, considered and appreciated" [9].

The dualities of the volunteer CHWs' role in the partnership being studied revealed the instrumentality of learning, as a CPBR construct, in determining partnership trust. Creating shared meaning and bridging differences through bidirectional sharing of knowledge between PASOs and the community, help volunteer CHWs use learning as a tool to develop partnership trust. For example, by actively seeking insight into the cultural nuances that their communities possess, CHWs increase their cultural competence, which then improves their delivery of programs and services. Consequently, through volunteer CHWs listening to and learning from their communities, PASOs can improve their services by then listening to and learning from the volunteer CHWs. A volunteer CHW expressed this,

"It's all about feedback. PASOs would not exist without the community, and unfortunately, the community still needs PASOs. Moreover, I say unfortunately because the ideal scenario would be that everyone would be able to solve their own problems, and that there would 


\section{Epidemiology International Journal}

not be such a need, and people could connect with the services that is often difficult to do. Because most of that is relatively impossible, you need an organization like PASOs to facilitate some of that learning and help people navigate those tough situations."

A volunteer CHW's position as both trustor and trustee exemplifies the importance of learning in promoting partnership trust; learning allows CHWs to bridge differences between their communities' actual needs and what PASOs perceives to be their needs. This knowledge bridging process promotes the development and sustainment of trust within the relationship.

When volunteer CHWs introduce new CPs to PASOs, it allows them the opportunity to bridge communication and safeguard against potential misconceptions by facilitating the integration of these new members. Furthermore, PASOs helps bridge misconceptions by accommodating new Latinx immigrants' needs and helping them learn about how systems work in their new environment, which helps advance in their skills and ability to procure resources for themselves. This is very important because as one CP mentioned, there is distrust and disunity within the communities due to fear; fear jeopardizes the sense of community among them. Therefore, partnership trust is nurtured when volunteer CHWs, and by extension PASOs, work to bridge differences with the communities they serve. This CP shared her appreciation for PASOs' work in bridging differences by saying,

"Listen, I like a place where they try to unify, and I would like to think that they do that here. That they truly help Hispanics, that they have programs to unify the community, because I repeat there is just a lot of distrust among the community. What came first the chicken or the egg? It is a good platform, which they are doing that, unifying, I want to think that is one of its principles, that they want to do that, but I know not everything is easy, and slowly they have been able to achieve that, and I want to be part of that."

OPs also take on very active roles as trustors in terms of learning and facilitating learning. The OPs that took part in the study aimed to create shared meaning through research and understanding and learning from communities' feedback. Some even worked to help staff CHWs advance their proficiency in research skills and research design which increased the staff's capacity to create shared meaning and bridge gaps through research.

Participation: Within the context of CBPR, participation functions as a facilitator to familiarity and sharing knowledge, skills and resources [9]. Participation is integral to developing partnership trust as it "requires physical and cognitive presence," which indicates care on the part of the stakeholders [9]. CPs' engagement in PASOs is suggestive of their trust in the organization. They share the knowledge and information they learn through PASOs with fellow community members and encourage others to get involved with PASOs and their research endeavors. This is suggestive of partnership trust because these actions indicate that they believe that interactions with PASOs positively benefit them. Furthermore, CPs also participate by entrusting information about their communities to PASOs, hence sharing knowledge with PASOs. One of the motivations behind sharing this information is to expand PASOs' familiarity with their communities so other members will be more willing to engage with the organization. As trustees, volunteer CHWs also work to engage community members with PASOs; while as trustors, they share their knowledge and resources with their communities to motivate that engagement. OPs share resources in the form of funding and expertise such as research expertise and by participating in PASOs' programs and activities. By providing funding to PASOs' various programs and participating in cultural competence training, they demonstrate their investment in PASOs' capacity and by extension their trustworthiness.

Effective teamwork requires respect, listening and understanding among those involved, and ultimately promotes participation. Teamwork allows PASOs' stakeholders to adopt a multifaceted approach to serving the different communities by considering diverse opinions and methods to tackle issues. CPs appreciate the multifaceted approaches, which then increases PASOs' perception of trustworthiness. Staff CHWs also work according to a system of checks and balances, which demonstrates the practice of respect and understanding. This system enables the approaches to community outreach to be distributed evenly, rather than being determined and driven by an individual person or leader. A staff CHW illustrated this by saying,

"We look at it together and then do sort of these checks and balance and ask how someone feels about it and what they were able to pull from the information we have. Sometimes we ask [the evaluator] to pull data information from our management system that may support an idea or something. And because we comb, try to comb things with you know a fine brush or whatever, then we usually arrive at decisions together where it's not like 'oh I just totally disagree,' because once we have gone through things like that, then the point is made and it's like 'oh I can see that [sic].' So most often we can all walk away feeling good about something versus someone feeling like 'oh that's just not going to work or whatever." 


\section{Epidemiology International Journal}

Commitment: Within the context of CBPR, commitment refers to the performance of shared values (taking action) and a demonstration of "being in this together" [9]. This variable is related to partnership trust because it calls for the enactment of responsibilities associated with trust. The cooperative efforts between the different stakeholders that have been previously discussed are demonstrative of performance of shared values and "being in this together;" for example, the act of exchanging information and resources among the stakeholders attest to their performance of shared values. PASOs' collaboration with the Centers for Disease Control (CDC) around the Zika virus and how it affected the Latinx community, required commitment from all the stakeholders involved, each contributing a role in the project to deal with this epidemic appropriately. It must be noted that the embracing of open communication across stakeholders, as well as PASOs' responsive nature and their system of checks and balances allow for enhanced commitment.

Multidirectional sharing of knowledge and attempts to build cultural competence across stakeholders also facilitates the ability to act with shared values and the sense of "being in this together." For example, a staff CHW shared that,

"PASOs is trying to create culturally tailored programs that can reach people in a way that makes sense, and the community brings forward those ideas on what culturally makes sense. And they'll tell us 'this doesn't work for us, this is offensive, this doesn't resonate with me and my culture' and so they really give us the feedback to be able to say we really have culturally appropriate programming [sic] one that people appreciate and are proud of making." PASOs' practice of partnering with natural leaders in the community sets the stage for performance of shared values.

Co-authorship involving staff CHWs and OPs in publications on PASOs' research, and the events hosted by PASOs, testify to the demonstration of commitment. However, there have been some challenges to commitment. Although OPs share knowledge and resources with PASOs, there have been instances where they are not updated on whether their recommendations were considered by program leadership. For example, one OP shared that,

"...I have given them some information but I do not know if they are going to make changes or not because I say 'okay we need more support-if you are doing this tell me how we can do it [sic].' I tell you; you have a good program that needs to be done bigger and faster to reach more people and it was like 'yes, but we only have one person and we want to do it well.' I do not say that you should change your qualities, but you need more people [sic]..."

CPs has also expressed concerns of lack of follow-up to their feedback. A CP shared that, they "ask for opinions (...) For example, [a staff CHW] would ask, 'how can we get the community to get closer to us'? Then I give my opinion and if they want, they can take it or not... Well I know they write them down. But not sure if they take them, and I don't really follow up with them." By failing to follow up with what is done with input, PASOs risks disturbing the perception of all parties "being in this together," which can negatively affect the trustors' disposition to trust the trustees in this relationship.

\section{Alignment with CBPR Principles}

As a construct, alignment with CBPR principles means that the execution of a research project or program pays great consideration to accepting the knowledge and experience of the communities involved as valid, and thus integrating their input into the research design [18]. This construct also refers to the degree to which equity was exercised within the partnership between the community and the research organization [9]. Furthermore, the wellbeing of the community is prioritized and a commitment to communityengaged research is demonstrated [9]. Therefore, the variables identified to review the meanings of this construct are genuine partnership and capacity building. These codes help determine the extent to which alignment with CBPR principles contributed to the development of trust among PASOs stakeholders.

Genuine partnership: Genuine partnership is a CBPR principle that relates to stakeholders learning from each other, which has been discussed at length in previous sections. By exchanging information and sharing knowledge across stakeholders, each member of the partnership is deemed to possess valuable knowledge and expertise to share with and teach one another. Open communication again assists the progress of this recognition. However, a language barrier might sometimes disturb this recognition. A volunteer CHW expressed how his limited English-speaking skills dissuaded him from participating in the decision-making process at PASOs, "I have been alone in a meeting with the whole team and it was in English. So, they translated., I kind of understand, but when I don't, I feel shame to talk. I have not been able to feel comfortable talking, to let my tongue go, so not yet."

Language barriers represent lost opportunities for insights from both partners to be recognized and shared in a partnership. Nevertheless, PASOs tries to make it as accommodating as possible for their stakeholders to share their knowledge and teach one another. PASOs' leadership makes genuine efforts to hear the input from their staff CHWs and involve them in the decision-making process at PASOs. Staff CHWs are also encouraged to learn from each other's thoughts, life experiences, ideas and opinions. A staff 


\section{Epidemiology International Journal}

CHW shared that PASOs' leadership "would always ask us our thoughts and opinions. [The Executive Director] always keeps us as part of the process." This point was reiterated when another staff CHW shared that, "she wants to hear from people what their thoughts are, why they think in such a way, definitely wants to hear from community members about what's happening and what's working and not working and so there is conversation." Similarly, a volunteer CHW expressed, "not only do I voice my opinions about the goals, but I also ask for their input about what the goals should be for them and their teams." While another volunteer CHW, when speaking on the working environment at PASOs shared that "each one speaks, each one expresses their point of view and explains why not, very calmly."

Capacity building: A key tenet of CBPR is the capacity to conduct research and gain proficiency in research skills $[9,18]$. Determining partnership trust through the influence of CBPR hinges on the stakeholders' capabilities to utilize and engage in CBPR. Through their involvement with PASOs, CPs were able to build their capacity to conduct investigations and research because PASOs afforded them opportunities to collect data and be involved in data analysis. A CP shared an example of her research experience with PASOs, "I helped design the study. I put that together with PASOs staff, and we collaborated with each other... I [did] interviews, which is what I do, and administer[ed] surveys also, that [was] fun, and recruit[ed] participants." Volunteer CHWs also built their capacity through data collection and facilitating workshops. They have also had opportunities to conduct action-oriented research that executes follow-up action in response to the results of PASOs-led investigations. Staff CHWs learn and develop research skills through OPs and collaboration with external parties. For example, a staff $\mathrm{CHW}$ shared that,

"When I participate in research with PASOs, I feel capable of deciding what to study, selecting a research question, helping design the study, and facilitating a focus group, interviews, surveys, recruiting participants, data, results. So, I feel like I participated in all these aspects. (...) For example, I might help to design a focus group tool but [an external party] would be the one who facilitated it and made sure that all the language because the questions were so crucial to be worded correctly. So [the external party] was really in charge of doing all of that kind of stuff."

\section{Size of the Group}

Preference for small groups: Chandlee Miller $M$ [20] found that levels of trust are higher among CPs in smaller groups than larger groups. Trust is better nurtured in a small group setting because it creates an environment that is less intimidating than large group settings. CPs, volunteer
CHWs and OPs all expressed their preference for working in small groups. As trustors, their individuality might be less threatened in a small group setting, and so, they will be more willing to participate in the group, thus nurturing their disposition to trust in the partnership. Moreover, small groups are more manageable and create environments that are more conducive to facilitating discussions on sensitive subjects that place CPs in a vulnerable position. A volunteer CHW echoed this notion by saying, "We have done meetings of, well the maximum has always been 10-12 people, and it is usually much easier to handle because the topics are delicate, I should say, sometimes I say more people, no. I do not think so. (...) I do not know, as it is more difficult to manage, more difficult to agree or get to a consensus."

Although staff CHWs shared that they preferred to work with smaller groups, they also stated that it depends on the program, because some will require larger audiences to ensure the well-being of communities. For example, when discussing the decision-making process a staff CHW said, "PASOs works so good at not just leaving it there sometimes but getting feedback if necessary and as needed from like a larger, like maybe from the community itself, like the target population we are trying to reach...do a needs assessment and really understanding that if we are going to engage in specific work it is that going to make sense to the community we are going to take it to." Meaning that if a decision needs to be made regarding the welfare of communities, then their input is necessary to ensure that the right services are provided; therefore, as much representation from communities is needed in the decision-making process as possible to increase the generalizability and applicability of the services offered by the organization.

\section{Formal Agreement}

In defining how formal agreements are used to address challenges faced in CBPR research, Wallerstein N, et al. [18] stated that these agreements "equalize partnership and promote mutual benefit" (p. 41). Formal agreements clarify the distinctive role of each stakeholder in the partnership, which subsequently helps to foster commitment to the partnership and research endeavor. Formal agreements also serve to build trust among the stakeholders involved. Thus, the variables used to operationalize this construct include verbal or written agreements for collaborative efforts, formal agreements on roles and responsibilities, contracts, and details on what the organization is going to do.

Verbal or written agreement for collaborative efforts: The perception of trustworthiness and the sustainability of partnership trust is reliant upon consistency and accountability. PASOs facilitate several potential collaborative efforts. Volunteer CHWs come to an agreement 


\section{Epidemiology International Journal}

with PASOs with the details of their role and what is expected of them. A volunteer CHW shared, "when I started working, I had to fill out some training paperwork, they are like checklists. You must learn about the mission, know the vision, but when I started as [a] volunteer everything was verbal, I did not have to sign anything. They would talk to me about what is PASOS; this is the organization, so that you get to understand better." Agreements among PASOs' stakeholders help to nurture trust because each party knows what to expect from the other and keeps each other accountable for the sake of the mission of the organization. A staff CHW provided an example of how a written agreement can facilitate the development of partnership trust between PASOs and CPs by saying,

"When I worked with my participants to offer the prenatal courses, I had them sign. There were four forms that were needed to be signed-about the care of children, authorizing a PASOs representative to provide the care, and then another one on a photo authorization. We explained that the pictures we took were for promotional materials, but we would never share their names to keep confidentiality and to not violate their trust. There was a third form regarding the educational nature of the program and that for real emergencies or further healthcare needs, they had to reach out to their doctor, because we were not staffed with doctors at PASOs. The last form was about letting them know that this was a course and a program, that their ideas and knowledge would be respected and that they were in a safe environment to express themselves."

Formal agreements on roles and responsibilities: Mutual understanding and compliance to this understanding by each stakeholder is essential to moving trust beyond a belief toward becoming a decision and the actual action of trusting. Even though their responsibilities are confined to what is detailed in their agreement, and their ability to continue working with PASOs depends on the contract, many volunteer CHWs expressed how often they go beyond their duties in giving assistance to members of their communities. For example, a volunteer CHW went beyond her duties to help a participant,

"There was a participant who had no job, four children, a specific situation and I had a bike and then [name] called me, and asked me,' [CHW name] what do we do about this man? Come with me. We will take some things to him.' I told [name] that I had a bicycle, if the man has no means of transportation the bike serves him. Then they knock on our door to help those people. In fact, there's a room with donations, books, food, little things, toys."

\section{Although volunteer CHWs approach their formal}

agreements with more flexibility, staff CHWs are more bound to their formal agreements, especially when funding is involved. Formal agreements between PASOs and funders stipulate the deliverables and identified outcomes that will be used as measures by which PASOs will be evaluated. In terms of collaborations with other organizations, the data showed that there was a clear idea of the roles and responsibilities of those involved in the research because of a memorandum of understanding (MOU) clarifying their specific roles. A staff CHW shared, "that is typically the language that we use with partners, not only our host agencies and our other sites but maybe clinics or other partners that are not our host agency because they're other partners in the community where we want to have a defined agreement about our work when we put community health work in their facility."

Additionally, CPs hold expectations of how PASOs helps communities, which is stated in a MOU, as described by a volunteer CHW, "It's like an explanation of the program, what procedures should be followed; they lay out everything for the participant. It benefits the participant, the staff, the Community Health Worker, and the organization." Lack of formal agreements could be partly responsible for excluding OPs from PASOs' program development, given that PASOs is not obligated to consider the recommendations that the partners suggest for programs. This can influence OPs' inclination to trust PASOs since this could be interpreted as not being listened to, which is an important factor in developing partnership trust.

\section{Discussion}

This study sought to investigate to what extent the CBPR constructs identified by Moore de Peralta, et al. [14] in the dimension of "situational, organizational and institutional constraints" of a modified version of the MMTM were indicatory of face-valid facilitators of trust in the partnership of various stakeholders with the PASOs organization. These constructs are supposed to function as catalysts that enable trustors (in this case PASOs' CPs, volunteer CHWs, and OPs) to trust trustees (PASOs' staff CHWs and leadership) by first believing that the trustees are trustworthy, then making the decision to trust, and then acting based on the trust developed. Findings of this study, conceptually, support Moore de Peralta, et al.'s [14,15] assertion that CBPR can promote partnership trust in community-engaged health research and programs, specifically in the case of PASOs. Based on this study's results the research team determined that the MMTM CBPR-related constructs showed conceptual face validity. Stakeholders' accounts support the hypothesis that CBPR is a trust-building process [9]. Based on the findings of this study, CBPR processes and methods, when implemented with fidelity, might have the potential to promote partnership trust, as evidenced among a sample of 


\section{Epidemiology International Journal}

Spanish and English-speaking stakeholders of this CBHO.

In the CCCI study, from which the primary data used in this secondary analysis was obtained, PASOs' stakeholders, including CPs, volunteer CHWs, and Ops, were considered trustors and staff CHWs and volunteer CHWs were considered trustees for CPs and OPs. Therefore, volunteer CHWs were both trustors and trustees based on the mediating position they hold between CPs and staff CHWS and OPs. This secondary analysis of CCCI data showed that volunteer CHWs and staff CHWs' perception of PASOs' trustworthiness could have been potentially influenced by selected CBPR processes and methods including, (a) facilitating the building of communities' research capacities through collaborative research efforts; (b) sustainability efforts such as empowering community leaders; and (c) facilitating effective communication practices like listening to, learning from and promoting the participation of the trustors.

Regarding our research question about whether those CBPR related processes and methods listed in the dimension of "situational, organizational and institutional constraints" of a modified version of the MMTM promote development of partnership trust, we found qualitative support that these CBPR related processes and methods contributed to PASOs being considered a trustworthy organization amongst their diverse stakeholders. PASOs stakeholders' narratives illustrated the relevance or importance of CBPR processes and methods in determining and fostering partnership trust. Narratives expressed by each stakeholder group suggested that the application of some of the CBPR principles helped PASOs engage with their stakeholders in such a way that stakeholders are "willing to render themselves vulnerable" to PASOs. Stakeholders demonstrate their trust in PASOs by means of sharing deeper insights to their needs (i.e., CPs), granting funding and other resources (i.e., OPs) and serving as liaisons between PASOs and the community (i.e., volunteer CHWs).

Volunteer CHWs were placed in a unique position where CPs' "willingness to render themselves vulnerable" to PASOs, was partially contingent upon volunteer CHWs "willingness to render themselves vulnerable" to PASOs. Volunteer CHWs serve as a bridge or connector between CPs and the organization, exemplifying what Lucero JE [9] described as "proxy trust." By acting as mediators, volunteer CHWs were able to build relationships that facilitated the development of partnership trust. For example, while relying on PASOs to assist them in advancing their proficiency in research, volunteer CHWs were also relied upon to build the research capacities of their communities. Another example entailed PASOs sharing the findings of their studies and other relevant information with volunteer CHWs, who then disseminated this information to their communities or used the information as insights that could help them improve the delivery of services to their communities.

Directed content analysis of qualitative data proved to be an effective method to approach theory validation. We addressed the research question that was asked in this study by using the predetermined codes based on the same literature from which Moore de Peralta, et al.'s [14,15] derived their CBPR principles included in the modified MMTM. The codes functioned as variables by which to measure the face validity of our modified version of Dietz and Den Hartog's MMTM.

\section{Implications and Recommendations}

This study addressed a gap in the literature regarding the relationship between CBPR and partnership trust in public health research $[12,13]$. Studies tend to focus on the transition from a state of historical distrust to trust in CBPRrelated public health research; however, how to go about developing and increasing partnership trust within this context is largely absent from the literature $[26,27]$. Findings of this study suggest that CBPR processes and methods can potentially facilitate or promote partnership trust; specifically related to the situational, organizational, and institutional constraints that influence research and programmatic collaborations between community stakeholders and CBHOs. Future studies related to community-engaged public health research and interventions would greatly benefit from exploring the influence of CBPR processes and methods in partnership trust's development.

This study is not exempt of limitations. It is possible that self-report in the CCCI original study would be biased and influenced by a Latinx cultural inclination to appear cooperative or "simpatía" [28]. Hopwood, et al. [29] noted that social desirability of Latinx participants in research could be culturally related. Members of Latinx cultures are more likely to exhibit collectivist tendencies than individualistic ones. Studies have found that an inverse relationship exists between cultural individualism and social desirability where less social desirability is associated with higher degrees of individualism. Given that the Latinx culture is more collectivist, social desirability tends to be higher in Latinx participants [29]. However, members of the research team, which conducted the preliminary studies that preceded this current study, employed the use of a self-completed survey to minimize social desirability bias. Other measures that the research team employed also included refraining from the use of names of people or organizations in the interviews, as well as, assuring participants that their anonymity will be maintained by allowing the PASOs representatives involved in the research team to only see aggregated data. 


\section{Epidemiology International Journal}

Another limitation is related to the recruitment of participants in one conveniently selected county of a southeastern state; thus, participants in this organization who live in other counties, as well as Latinx residents in other US states may have different beliefs about and perceptions of CBPR and partnership trust that will not be represented by the sample's responses. Therefore, the diversity among Latinx subcultures and diversity within Latinx language was not fully represented in this study. In addition, the research team member who took the lead in the secondary analysis of the CCCI data for this study was not involved in the design and implementation of the two preliminary studies conducted by Moore de Peralta, et al. [14,15], nor did she participate in the transcription of the interview data. Transcribing interview data is an important part of data analysis because the researcher can take note of how other verbal cues such as tone, speed, pauses, etc. could influence the interpretation of the data [30]. These two limitations could have potentially resulted in inconsistent data interpretations. However, authors attempted to address these limitations by the subsequent review of coding structures performed by coauthors who were active in the design, implementation and analyses of these preliminary studies.

\section{Conclusion}

Selected constructs were used to operationalize CBPR processes and methods as potential determinants of partnership trust and were subjected to face validity testing. These constructs are included in the dimension of "situational, institutional and organizational constraints" of the MMTM [16] and were found to hold face validity when operationalized through our coding by using the CCCI study participants' accounts and the research team's interpretations. The codes allowed the researchers of this study to account for the degree to which the data provided by the stakeholders, via the CCCI transcripts, were demonstrative and indicative of the role CBPR has in promoting and sustaining partnership trust among a health promotion network which included a community-based health promotion organization, its organizational partners, and members of the communities they serve. In this study, directed content analysis of qualitative data was found to be an effective method to approach theory validation. The face validity testing conducted in this study, by means of performing a deductive content analysis, produced supporting evidence that substantiates the potential of CBPR processes and methods as facilitators or determinants of partnership trust.

\section{References}

1. (2018) Hispanic Heritage Month. United States Census Bureau.
2. (2017) Health of Hispanic or Latino Population. FastStats. CDC.

3. (2015) ¡A la Buena Salud! - To Good Health! Hispanic Health. CDC.

4. (2019) Hispanic/Latino Americans. Office of Minority Health.

5. Velasco Mondragon E, Jimenez A, Palladino Davis AG, Davis D, Escamilla Cejudo JA (2016) Hispanic health in the USA: a scoping review of the literature. Public Health Rev 37(31).

6. Michael YL, Farquhar SA, Wiggins N, Mandy k Green (2008) Findings from a Community-based Participatory Prevention Research Intervention Designed to Increase Social Capital in Latino and African American Communities. J Immigr Minor Health 10(3): 281-289.

7. Wallerstein NB, Duran B (2006) Using community-based participatory research to address health disparities. Health Promot Pract 7(3): 312-323.

8. Sandoval JA, Lucero J, Oetzel J, Magdalena A, Lorenda $B$, et al. (2012) Process and outcome constructs for evaluating community-based participatory research projects: a matrix of existing measures. Health Educ Res 27(4): 680-690.

9. Lucero JE (2013) Trust as an ethical construct in community-based participatory research partnerships. (Doctoral dissertation). Albuquerque: University of New Mexico.

10. Tumiel-Berhalter L, Kahn L, Watkins R, Melanie Goehle, Carrie Meyer (2011) The implementation of Good for the Neighborhood: a participatory community health program model in four minority underserved communities. J Community Health 36(4): 669-674.

11. (2008) Center for Disease Control and Prevention [CDC]. CDC prevention research centers' partnership trust tool survey.

12. Calnan M, Rowe R (2006) Researching trust relations in health care: conceptual and methodological challengesintroduction. J Health Organ Manag 20(5): 349-358.

13. (2013) Funding Opportunity Announcement (PA-13209). Department of Health and Human Services.

14. Moore de Peralta A, Smithwick J, Torres ME (2020) Perceptions and determinants of partnership trust in the context of Community-Based Participatory Research. J. Health Dispar Res Pract 13(1): 67 - 95. 


\section{Epidemiology International Journal}

15. Moore de Peralta A, Prieto Rosas V, Smithwick J, Timmons $\mathrm{S}$, Torres ME (In Press) A contribution to measure partnership trust in community-based participatory research and interventions with Latinx communities in the United States. Health Promot Pract.

16. Dietz G, Den Hartog, Deanne N (2006) Measuring trust inside organizations. Pers Rev 35(5): 557-588.

17. Belone L, Lucero JE, Duran B, Greg Tafoya, Elizabeth A Baker, et al. (2016) Community-Based Participatory Research Conceptual Model: Community Partner Consultation and Face Validity. Qual Health Res 26(1): 117-135.

18. Wallerstein N, Duran B (2010) Community-based participatory research contributions to intervention research: The intersection of science and practice to improve health equity. Am J Public Health 100(1): 40-46.

19. Costa AC, Anderson N (2011) Measuring trust in teams: Development and validation of a multifaceted measure of formative and reflective indicators of team trust. Eur J Work Organ Psychol 20(1): 119-154.

20. Chandlee Miller M (2012) Measuring trust in communitybased and congregation-based groups in South Carolina: A psychometric analysis and exploration of trust, group characteristics, and health.

21. Oetzel JG (2009) Intercultural communication: A layered approach New York: Pearson Education.

22. Mandelblatt JS, Ramsey SD, Lieu TA, Phelps CE (2017) Evaluating Frameworks That Provide Value Measures for Health Care Interventions. Value Health 20(2): 185192.
23. Willke RJ, Neumann PJ, Garrison LP Jr, Ramsey SD (2018) Review of Recent US Value Frameworks-A Health Economics Approach: An ISPOR Special Task Force Report [6]. Value Health 21(2):155-160.

24. McDonald KM, Sundaram V, Bravata DM, Robyn Lewis, Nancy Lin, et al. (2007) Closing the Quality Gap: A Critical Analysis of Quality Improvement Strategies (Vol. 7: Care Coordination). Rockville (MD): Agency for Healthcare Research and Quality (US) 5(9.7).

25. Willis GB (2011) Cognitive Interviewing: A Tool for Improving Questionnaire Design. Thousand Oaks: Sage.

26. Hsieh H, Shannon SE (2005) Three approaches to qualitative content analysis. Qual Health Res 15(9): 1277-1288.

27. Metzler MM, Higgins DL, Beeker CG, Nicholas F, Paula M Lantz, et al. (2003) Addressing urban health in Detroit, New York City, and Seattle through community-based participatory research partnerships. Am J Public Health 93(5): 803-811.

28. Arredondo EM, Pollak K, Costanzo PR (2008) Evaluating a stage model in predicting monolingual Spanishspeaking Latinas' cervical cancer screening practices: The role of psychosocial and cultural predictors. Health Educ Behav 35(6): 791-805.

29. Hopwood CJ, Flato CG, Ambwani S, Garland BH, Morey LC (2009) A comparison of Latino and Anglo socially desirable responding. J Clin Psychol 65(7): 769-780.

30. Hesse Biber SN (2017) The Practice of Qualitative Research: Engaging Students in the Research Process. $3^{\text {rd }}$ (Edn.), SAGE, Los Angeles. 